\title{
A Novel Train-to-Train Communication Model Design Based on Multihop in High-Speed Railway
}

\author{
Pengyu Liu, ${ }^{1}$ Bo Ai, ${ }^{1}$ Zhangdui Zhong, ${ }^{1}$ and Xiaojuan Zhou' \\ ${ }^{1}$ State Key Laboratory of Rail Traffic Control and Safety, Beijing Jiaotong University, Beijing 100044, China \\ ${ }^{2}$ Department of Communication Engineering, University of Armed Police Force Engineering, Xian 710086, China
}

Correspondence should be addressed to Bo Ai, aibo@ieee.org

Received 22 July 2012; Accepted 16 October 2012

Academic Editor: César Briso Rodríguez

Copyright ( $) 2012$ Pengyu Liu et al. This is an open access article distributed under the Creative Commons Attribution License, which permits unrestricted use, distribution, and reproduction in any medium, provided the original work is properly cited.

\begin{abstract}
Railway telematics applications are currently attracting attention and are under intense research. Reliable railway telematics applications increasingly tend to require a subsidiary means to help existent control system make train operation safer and more efficient. Since 2006, train-to-train communication has been studied to respond to such requirements. A key characteristic of train-to-train communication is that operation control to avoid possible accidents is conducted among trains without help of a base station. This paper proposes a novel train-to-train communication model in a physical layer based on multihop and cooperation, taking a high-speed railway propagation channel into account. The mechanism of this model lies in the idea that a source train uses trains on other tracks as relays to transmit signals to destination train on the same track. Based on occurrence of these potential relays, such mechanism can be divided into three cases. In each case, BER is applied to evaluate properties of the proposed communication model. Simulation results show that BER of the train-to-train communication model decreases to $10^{-6}$ when SNR is $10 \mathrm{~dB}$ and that the minimum receiving voltage of this model is $-84 \mathrm{dBm}$, which is $8 \mathrm{dBm}$ lower than the standards established by the International Union of Railways (UIC) in a high-speed railway scenario.
\end{abstract}

\section{Introduction}

Railways are a powerful transportation systems which exert significant influence in supporting development of economies. Safety concerns in railways are attracting an increasing amount of attention at present, because the railways have assumed an increased responsibility for safeguarding the personal and property security. Railway accidents generally lead to serious consequences-loss of lives and property. These phenomena directly motivate the researchers to concentrate more on various systems for railway safety. The mainstream technique of railway safety in China is the Chinese Train Control System Level 3 (CTCS-3) based on the Global System for Mobile Communication for Railways (GSM-R) which acts as a radio interface to link trains with control center to exchange safety messages; this system ensures that trains are monitored by a real-time device and that they operate at a certain safe distance from each other [1].

It cannot be denied that the CTCS-3 system has proved to be an accurate technique for positioning and also provides a rapid exchange of motion state and control messages. However, according to statistics provided by the American Federal Railroad Administration (FRA) in the United States, about 8221 accidents threaten the passengers' personal safety in the past four years [2]. This is because a train driver could only be informed about potential collisions by an operation center. If the operation center fails to transmit control messages in an emergency, an accident will inevitably occur. Therefore, it is imperative to develop a novel technique to assist existing system to make control of train operation safer and more accurate. This technique allows the train conductors to keep updated with accurate information of traffic conditions in their vicinity [3]. On the basis of intertrain multihop communication, the train-to-train communication aims at detecting a potential collision and then broadcast prewarning messages to other trains on the same and neighboring tracks. When a control center system detects potential accidents, the train-to-train communication acts in an assisting role to immediately propagate messages to other trains and provide potential solutions to the driver to avoid 
danger. Furthermore, its application also reduces outlays on infrastructure maintenance for base stations [4].

\section{Related Work}

In recent years, research on train-to-train communication has been carried out by several organizations, including the German Aerospace Center (DLR). Reference [5] discusses communication link design such as maximum data rate, frequency selection, and channel model, while [6] describes an overview of the state of the art in collision avoidance related to transportation systems for maritime, aircraft, and road transportation, and the RCAS is introduced. Reference [7] proposes a channel model for direct train-to-train communication appropriate for the $400 \mathrm{MHz}$ band, and [8] presents analyses and results of a comprehensive measurement campaign investigating the propagation channel in case of direct communication between railway vehicles.

Though the RCAS has undergone some progress in physical-layer design, it only supports train operation velocity of lower than $200 \mathrm{Km} / \mathrm{h}$, which is not applicable to a highspeed railway. Generally, the velocity of a high-speed railway train is up to $360 \mathrm{Km} / \mathrm{h}$. In this case, safety distance among trains is $10 \mathrm{Km}$ [9], which will result in severe path loss and poor receiving signal quality if two trains on the same track perform direct communication. The BER of the receiving signal is about 0.5 .

To solve this problem, in this paper, we propose a trainto-train communication model in the physical layer based on multihop. In order to make up a poor receiving signal resulting from path loss, the multihop mechanism in which the source train uses trains operating on other tracks as relays to transmit signals to a destination train on the same track is adopted. This mechanism can be divided into three cases based on occurrence of other relay trains. A presumption can be made that an arrival procedure of a train follows the Poisson distribution and the arrival procedure is a negative exponent [10]. In contrast to the physical-layer model research in other papers $[5,6]$, the proposed trainto-train communication model, introducing OFDM and MIMO techniques, realizes intertrain adhoc communication based on the Poisson process in the high-speed railway scenarios.

The rest of this paper is organized as follows. Section 3 gives a description of the proposed train-to-train communication model based on multihop, and Section 4 derives BER expressions of three cooperative conditions. In Section 5, the parameter selection of the train-to-train model is discussed. Section 6 shows the simulation results of this model, and this paper is concluded in Section 7.

\section{Proposed Train-to-Train Communication Model}

The train-to-train communication model is presented in Figure 1, where the $S, R 1$, and $D$ represent a source terminal, a fixed-occurring relay terminal, and a destination terminal, respectively. The $R 1$ is the train that meets $S$ on another rail track, and the $R 2$ and $R 3$, respectively, represent the

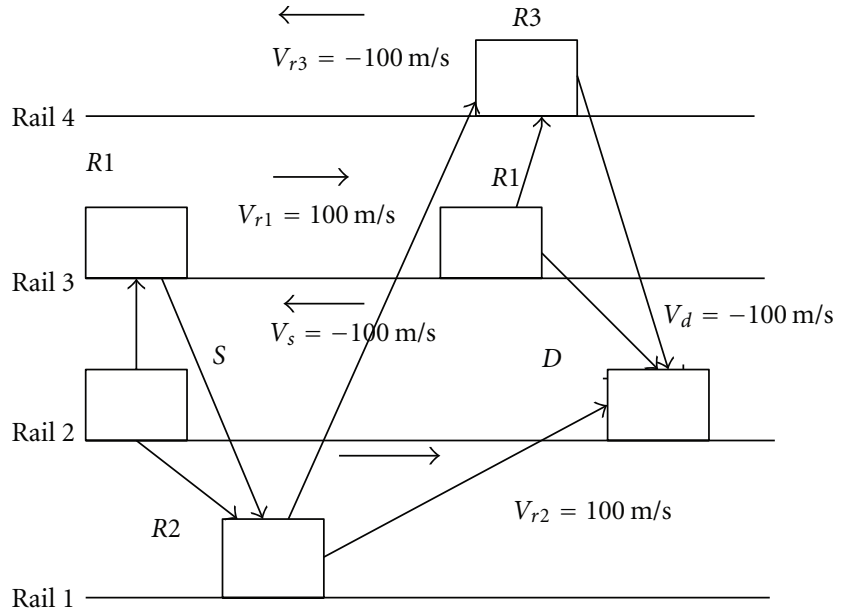

FIGURE 1: Communication mechanism of train-to-train model.

first possible-occurring relay terminal and the third possibleoccurring relay terminal. The average operating velocities of the $R 1$ and $R 2$ are $100 \mathrm{~m} / \mathrm{s}$, and those of $D$ and $R 3$ are -100 m/s. Rail 1, Rail 2, Rail 3, and Rail 4 represent four parallel rail tracks.

The communication mechanism of this model can be discussed under three cases based on the Poisson process. In the Poisson process, let sequence $\left\{X_{n}, n \geq 1\right\}$ denote the time interval between the $(n-1)$ th and the $n$th event, called the sequence of interarrival times, and $X_{n}$ follows an exponential distribution. If a certain event definitely occurs, $X_{n}$ will be uniformly distributed in the time interval $T$ [11]. The occurrence of potential relays follows the Poisson process. Due to the concept put forward in China that the train flow density of trains in a high-speed railway network tends to be the same as that of buses in road transportation [12], there is no doubt that a two-train meet among different tracks will always exist. These cases are described below.

3.1. Case $I$. The $S$ transmits signals to the $R 1$ on different rail tracks when they meet each other. At the same time, the $S$ searches for the potential relay $R 2$ on a neighboring rail track 1 . If the $R 2$ is not found, $R 1$ will keep broadcasting messages within its communication coverage $(6 \mathrm{Km})$ [5], and if it receives a response from $D$, their communication link will be held and the transmission between them is performed.

3.2. Case II. If the $R 2$ is searched, the $S$ and $R 1$ simultaneously transmit the signals to the $R 2$. The $R 2$ also performs the search of the potential relay $R 3$. If the $R 3$ does not exist, it will operate for some seconds until the distance between $R 2$ and $D$ is within a communication range. Finally, the $R 2$ and $R 1$, acting as two relays of the source, will transmit the signals to the destination terminal.

3.3. Case III. If the $R 3$ exists, it will receive the signals from the $R 2$ and $R 1$ and then combine them. Finally, the $R 3, R 2$, and $R 1$, as three relays of the source, will forward the signals to the destination terminal. 


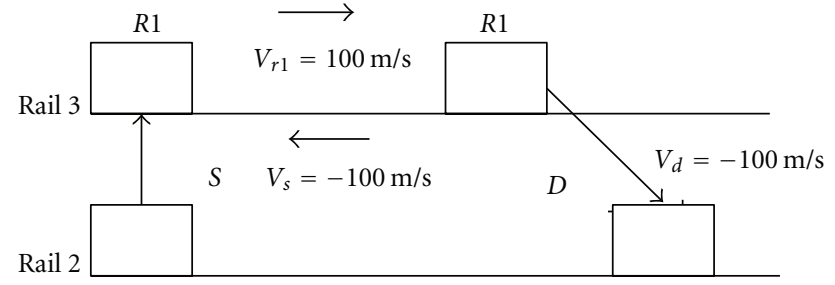

FIgURe 2: Communication model of Case I.

Under these cases, this paper respectively analyzes the reliability of the train-to-train communication model using the performance index BER.

\section{Analysis of Train-to-Train Communication Model}

4.1. Case I. Figure 2 describes the train-to-train communication model of Case I. The source $S$ transmits the signals to the $R 1$ as they meet each other. Total power is $E$, and the transmit signals are denoted as follows:

$$
\begin{aligned}
& \mathbf{X}_{S, R 1,2 k+1}(n)=\left(x_{S, R 1,2 k+1}(n) ;\right. \\
& n=\left\{N-N_{\mathrm{CP}}+1, \ldots, N, 1,2, \ldots, N\right\}, \\
&\left.k=\left\{0,2, \ldots, \frac{M-2}{2}\right\}\right), \\
& \mathbf{X}_{S, R 1,2 k+1}^{*}(n)=\left(x_{S, R 1,2 k+1}^{*}(n) ;\right. \\
& n=\left\{N-N_{\mathrm{CP}}+1, \ldots, N, 1,2, \ldots, N\right\}, \\
&\left.k=\left\{0,2, \ldots, \frac{M-2}{2}\right\}\right), \\
& \mathbf{X}_{S, R 1,2 k+2}(n)=\left(x_{S, R 1,2 k+2}(n) ;\right. \\
& n=\left\{N-N_{\mathrm{CP}}+1, \ldots, N, 1,2, \ldots, N\right\}, \\
&\left.k=\left\{0,2, \ldots, \frac{M-2}{2}\right\}\right), \\
&-\mathbf{X}_{S, R 1,2 k+2}^{*}(n)=\left(-x_{S, R 1,2 k+2}^{*}(n) ;\right. \\
& n=\left\{N-N_{\mathrm{CP}}+1, \ldots, N, 1,2, \ldots, N\right\}, \\
&\left.k=\left\{0,2, \ldots, \frac{M-2}{2}\right\}\right) .
\end{aligned}
$$

The $\quad \mathbf{X}_{S, R 1,2 k+1}(n), \quad \mathbf{X}_{S, R 1,2 k+2}(n), \quad \mathbf{X}_{S, R 1,2 k+1}^{*}(n), \quad$ and $-\mathbf{X}_{S, R 1,2 k+2}^{*}(n)$ denote two different transmitting OFDM symbols and their conjugate forms. The $x_{S, R 1,2 k+1}(n)$, $x_{S, R 1,2 k+1}^{*}(n), x_{S, R 1,2 k+2}(n), x_{S, R 1,2 k+2}(n)$, and $-x_{S, R 1,2 k+2}^{*}(n)$ are the Fast Fourier Transforms (FFT) of the Quadrature Phase Shift Keying (QPSK) modulation symbol in each subcarrier of the OFDM symbol. In OFDM modulation, $n$ represents the sequence number of the subcarrier. $N$ and $N_{\mathrm{CP}}$, are, respectively, the total number of subcarriers and the length of the cyclic prefix. $M$ is the number of transmit OFDM symbols.

Considering Alamouti coding [13], $\mathbf{X}_{S, R 1}$ denotes the transmit signals at the transmitter with two antennas (antenna 1 and antenna 2) and is expressed as below:

$$
\mathbf{X}_{S, R 1}=\left[\begin{array}{cc}
\mathbf{X}_{S, R 1,2 k+1}(n) & \mathbf{X}_{S, R 1,2 k+2}(n) \\
-\mathbf{X}_{S, R 1,2 k+2}^{*}(n) & \mathbf{X}_{S, R 1,2 k+1}^{*}(n)
\end{array}\right]
$$

Having taken multipaths and the Doppler effect into account, the impulse response of a channel can be written as

$$
\begin{aligned}
& \mathbf{H}_{1, S, R 1, l}=\left(\mathrm{a}_{l} \delta\left(n-n_{l}\right) \exp \left(2 \pi T_{s} f_{d}\left(n-n_{l}\right)\right) ;\right. \\
&\left.n=\left\{N-N_{\mathrm{CP}}+1, \ldots, N, 1,2, \ldots, N\right\}\right), \\
& \mathbf{H}_{2, S, R 1, l}=\left(\mathrm{a}_{l} \delta\left(n-n_{l}\right) \exp \left(2 \pi T_{s} f_{d}\left(n-n_{l}\right)\right),\right. \\
&\left.n=\left\{N-N_{\mathrm{CP}}+1, \ldots, N, 1,2, \ldots, N\right\}\right)
\end{aligned}
$$

$\mathbf{H}_{1, S, R 1, l}$ and $\mathbf{H}_{2, S, R 1, l}$ are channel impulse responses of a certain single path from the transmitter to the receiver with a $2 \times 2$ MIMO. $l$ denotes the number of multipaths, $f_{d}$ is the maximum Doppler frequency shift, $n_{l}$ is the time delay of each path, and $T_{S}$ is the sampling interval.

The $\mathbf{Y}_{1, S, R 1,2 k+1, l},-\mathbf{Y}_{1, S, R 1,2 k+2, l}^{*}, \mathbf{Y}_{2, S, R 1,2 k+2, l}, \quad$ and $\mathbf{Y}_{1, S, R 1,2 k+1, l}^{*}$ are the receiving vectors at the receiver for two antennas, and they can be derived as follows:

$$
\begin{aligned}
& {\left[\begin{array}{cc}
\mathbf{Y}_{1, S, R 1,2 k+1, l} & \mathbf{Y}_{2, S, R 1,2 k+1, l} \\
-\mathbf{Y}_{1, S, R 1,2 k+2, l}^{*} & -\mathbf{Y}_{2, S, R 1,2 k+2, l}^{*}
\end{array}\right]} \\
& =\sqrt{E / 2}\left[\begin{array}{cc}
\mathbf{X}_{S, R 1,2 k+1} \odot \mathbf{H}_{1, S, R 1, l} & \mathbf{X}_{S, R 1,2 k+1} \odot \mathbf{H}_{2, S, R 1, l} \\
-\mathbf{X}_{S, R 1,2 k+2}^{*} \odot \mathbf{H}_{1, S, R 1, l} & -\mathbf{X}_{S, R 1,2 k+2}^{*} \odot \mathbf{H}_{2, S, R 1, l}^{*}
\end{array}\right] \\
& +\left[\begin{array}{ll}
\mathbf{N}_{1, S, R 1,2 k+1, l} & \mathbf{N}_{2, S, R 1,2 k+1, l} \\
\mathbf{N}_{1, S, R 1,2 k+2, l}^{\prime} & \mathbf{N}_{2, S, R 1,2 k+2, l}^{\prime}
\end{array}\right], \\
& {\left[\begin{array}{lll}
\mathbf{Y}_{1, S, R 1,2 k+2, l} & \mathbf{Y}_{2, S, R 1,2 k+2, l} \\
\mathbf{Y}_{1, S, R 1,2 k+1, l}^{*} & \mathbf{Y}_{2, S, R 1,2 k+1, l}^{*}
\end{array}\right]} \\
& =\sqrt{E / 2}\left[\begin{array}{ll}
\mathbf{X}_{S, R 1,2 k+2} \odot \mathbf{H}_{1, S, R 1, l} & \mathbf{X}_{S, R 1,2 k+2} \odot \mathbf{H}_{2, S, R 1, l} \\
\mathbf{X}_{S, R 1,2 k+1}^{*} \odot \mathbf{H}_{1, S, R 1, l} & \mathbf{X}_{S, R 1,2 k+1}^{*} \odot \mathbf{H}_{2, S, R 1, l}^{*}
\end{array}\right] \\
& +\left[\begin{array}{lll}
\mathbf{N}_{1, S, R 1,2 k+2, l} & \mathbf{N}_{2, S, R 1,2 k+2, l} \\
\mathbf{N}_{1, S, R 1,2 k+1, l}^{\prime} & \mathbf{N}_{2, S, R 1,2 k+1, l}^{\prime}
\end{array}\right] . \\
& \mathbf{N}_{1, S, R 1,2 k+1, l,} \quad \mathbf{N}_{2, S, R 1,2 k+1, l,} \quad \mathbf{N}_{1, S, R 1,2 k+2, l}^{\prime} \quad \mathbf{N}_{2, S, R 1,2 k+2, l}^{\prime}, \\
& \mathbf{N}_{1, S, R 1,2 k+2, l}, \mathbf{N}_{2, S, R 1,2 k+2, l}, \mathbf{N}_{1, S, R 1,2 k+1, l}^{\prime} \text { and } \mathbf{N}_{2, S, R 1,2 k+1, l}^{\prime} \text { are the }
\end{aligned}
$$
Additive White Gaussian Noise (AWGN) with a zero mean

$$
\begin{aligned}
& \mathbf{Y}_{1, S, R 1,2 k+1}=\sum_{l=1}^{L} \mathbf{Y}_{1, S, R 1,2 k+1, l}, \\
& \mathbf{Y}_{2, S, R 1,2 k+1}=\sum_{l=1}^{L} \mathbf{Y}_{2, S, R 1,2 k+1, l}, \\
& \mathbf{Y}_{1, S, R 1,2 k+2}=\sum_{l=1}^{L} \mathbf{Y}_{1, S, R 1,2 k+2, l},
\end{aligned}
$$




$$
\begin{gathered}
\mathbf{Y}_{1, S, R 1,2 k+2}=\sum_{l=1}^{L} \mathbf{Y}_{1, S, R 1,2 k+2, l}, \\
\mathbf{Y}_{S, R 1,2 k+1}=\mathbf{Y}_{1, S, R 1,2 k+1}+\mathbf{Y}_{2, S, R 1,2 k+1}, \\
\mathbf{Y}_{S, R 1,2 k+2}=\mathbf{Y}_{1, S, R 1,2 k+2}+\mathbf{Y}_{2, S, R 1,2 k+2} .
\end{gathered}
$$

$L$ is the total quantity of multipaths. By processing at the receiver, $\mathbf{Y}_{S, R 1,2 k+1}$ and $\mathbf{Y}_{S, R 1,2 k+2}$ are the final receiving vectors with signals of $L$ paths added together.

Using the modified $L S$ channel estimation, $\mathbf{H}_{S, R 1,2 k+1}^{\prime}$ and $\mathbf{H}_{S, R 1,2 k+2}^{\prime}$ are obtained to estimate the original source signals:

$$
\begin{aligned}
& \mathbf{X}_{S, R 1,2 k+1}^{\prime}=\mathbf{H}_{S, R 1,2 k+1}^{\prime-1} \odot \mathbf{Y}_{S, R 1,2 k+1} \\
& \mathbf{X}_{S, R 1,2 k+2}^{\prime}=\mathbf{H}_{S, R 1,2 k+2}^{\prime-1} \odot \mathbf{Y}_{S, R 1,2 k+2}
\end{aligned}
$$

$\mathbf{X}_{S, R 1,2 k+1}^{\prime}$ and $\mathbf{X}_{S, R 1,2 k+2}^{\prime}$ denote the estimation source signals, and the SNRs $\Upsilon_{R 1,2 k+1}$ and $\Upsilon_{R 1,2 k+2}$ at the relay can be written as

$$
\begin{aligned}
& \Upsilon_{R 1,2 k+1}=\frac{E}{4 N_{0} N}\left\|\mathbf{X}_{S, R 1,2 k+1}^{\prime}\right\|^{2}, \\
& \Upsilon_{R 1,2 k+2}=\frac{E}{4 N_{0} N}\left\|\mathbf{X}_{S, R 1,2 k+2}^{\prime}\right\|^{2} .
\end{aligned}
$$

At the relay $R 1$, the $\mathbf{X}_{S, R 1,2 k+1}^{\prime}$ and $\mathbf{X}_{S, R 1,2 k+2}^{\prime}$ are demodulated and then encoded again using QPSK and OFDM modulation to prepare them to be forwarded.

From the $R 1$ to the destination, the transmitting signals $\mathbf{X}_{S, R 1,2 k+1}^{\prime}$ and $\mathbf{X}_{S, R 1,2 k+2}^{\prime}$ have experienced the same process as $\mathbf{X}_{S, R 1,2 k+1}$ and $\mathbf{X}_{S, R 1,2 k+2}$.

$\mathbf{X}_{R 1, D, 2 k+1}^{\prime}$ and $\mathbf{X}_{R 1, D, 2 k+2}^{\prime}$ are the estimation relay signals, and the SNRs $\Upsilon_{D, 2 k+1}$ and $\Upsilon_{D, 2 k+2}$ at the relay can be written as

$$
\begin{aligned}
& \Upsilon_{D, 2 k+1}=\frac{E}{4 N_{0} N}\left\|\mathbf{X}_{R 1, D, 2 k+1}^{\prime}\right\|^{2}, \\
& \Upsilon_{D, 2 k+2}=\frac{E}{4 N_{0} N}\left\|\mathbf{X}_{R 1, D, 2 k+2}^{\prime}\right\|^{2} .
\end{aligned}
$$
[13]:

$P_{e}$ is the BER of the QPSK and can be expressed as below

$$
P_{e}=Q(\sqrt{2 \Upsilon})
$$

where $\Upsilon$ is the SNR.

The BER of the relay strategy Decode and Forward (DF) can be written as [14]

$$
P_{e}=Q(\sqrt{2 \Upsilon})+Q(\sqrt{2 \Upsilon})-2 Q(\sqrt{2 \Upsilon}) Q(\sqrt{2 \Upsilon}) .
$$

$P_{e, 2 k+1}$ and $P_{e, 2 k+2}$ are the BER of the $(2 k+1)$ th and $(2 k+$ 2)th OFDM symbols:

$$
\begin{aligned}
P_{e, 2 k+1}= & Q\left(\sqrt{2 \Upsilon_{R 1,2 k+1}}\right)+Q\left(\sqrt{2 \Upsilon_{D, 2 k+1}}\right) \\
& -2 Q\left(\sqrt{2 \Upsilon_{R 1,2 k+1}}\right) Q\left(\sqrt{2 \Upsilon_{D, 2 k+1}}\right) .
\end{aligned}
$$

The channel encoding is applied to enforce the error detection and correction at the receiver, and considering adoption interleaving and a $(7,4)$ cyclic code, the destination's BERs of the $(2 k+1)$ th and $(2 k+2)$ th OFDM symbols can be

$$
\begin{aligned}
& P_{e, \mathrm{I}, 2 k+1}=\sum_{m=t+1}^{n} C_{n}^{m}\left(P_{e, 2 k+1}\right)^{m}\left(1-P_{e, 2 k+1}\right)^{n-m}, \\
& P_{e, \mathrm{I}, 2 k+2}=\sum_{m=t+1}^{n} C_{n}^{m}\left(P_{e, 2 k+2}\right)^{m}\left(1-P_{e, 2 k+2}\right)^{n-m} .
\end{aligned}
$$

$P_{e, \mathrm{I}, 2 k+1}$ and $P_{e, \mathrm{I}, 2 k+2}$ are the BERs of the $(2 k+1)$ th and $(2 k+2)$ th OFDM symbols at the destination terminal, $t$ is the number of errors which can be corrected, and $n$ is the total number of bit streams.

4.2. Case II. The distance between source and destination terminals is $10 \mathrm{Km}$, which is out of the set communication range $(6 \mathrm{Km})$. It is therefore feasible to communicate with the destination terminal via $R 2$ within the communication range on the neighboring track. Because the average velocity of the $R 2$ is $100 \mathrm{~m} / \mathrm{s}$, the operation time interval in the communication range is $50 \mathrm{~s}$, considering $1 \mathrm{~km}$ as the reserve margin.

Figure 3 gives a description of the train-to-train communication model of Case II. At time slot $m$, the source transmits the signals to $R 1$, when they meet each other. The transmit signals are the same as those of Case I. At the same time, $S$ searches $R 2$ on the neighboring track. The occurrence of the $R 2$ is on the basis of the Poisson process. Supposing the occurrence of $R 2$ is event 1 , denoted as $S 1$, the coming of $S 1$ follows exponential distribution within the time interval $T$ of which the average strength $\lambda$ is $180 \mathrm{~s}$ [9]. The coming probability $P_{S 1}$ of event $S 1$ in the time interval $T$ can be obtained as follows:

$$
\begin{gathered}
T=\frac{10,000 \mathrm{~m}}{(100 \mathrm{~m} / \mathrm{s})-(-100 \mathrm{~m} / \mathrm{s})}=50 \mathrm{~s}, \\
P_{S 1}=1-\left.\exp \left(-\frac{t}{\lambda}\right)\right|_{T=50 \mathrm{~s}, \lambda=180 \mathrm{~s}}=0.2425 .
\end{gathered}
$$

When the event $S 1$ is determinately occurring, its distribution in the $50 \mathrm{~s}$ is uniform $U(0,50)$.

At time slot $m+1$, the source and $R 1$ simultaneously transmit the signals to the $R 2$, and the transmit signals $\mathbf{X}_{S, R 2}(n)$ and $\mathbf{X}_{R 1, R 2}(n)$ from $S$ and $R 2$ are written as

$$
\begin{aligned}
\mathbf{X}_{S, R 2}(n)= & \left(x_{S, R 1}(n) ;\right. \\
& \left.n=\left\{N-N_{C P}+1, \ldots, N, 1,2, \ldots, N\right\}\right), \\
\mathbf{X}_{R 1, R 2}(n)= & \left(x_{S, R 1}(n) ;\right. \\
& \left.n=\left\{N-N_{C P}+1, \ldots, N, 1,2, \ldots, N\right\}\right) .
\end{aligned}
$$

Like Case I, the transmitter adopts OFDM and Alamouti coding technique. The transmit signals are transmitted with multipath and Doppler effects and are processed by the relay $R 2$ to acquire the estimation signals. 


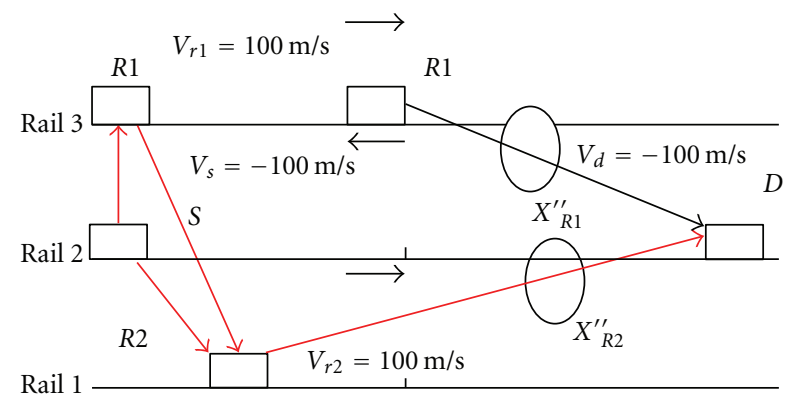

FIgURE 3: Communication model of Case II.

After decoding and reencoding the receiving signals, the $R 2$ operates for some seconds until the distance between $R 2$ and $D$ is within the communication range.

$R 2$ and $R 1$, as two relays of the source, jointly transmit the estimation signals $\mathbf{X}_{R 1}^{\prime}$ and $\mathbf{X}_{R 2}^{\prime}$ to the destination terminal, on the assumption that the relays $R 2$ and $R 1$ coordinate perfectly. The estimation signals at the destination terminal are $\mathbf{X}_{R 1}^{\prime \prime}, \mathbf{X}_{R 2}^{\prime \prime}$ and the SNRs $\Upsilon_{R 1}$ and $\Upsilon_{R 2}$ at the destination terminal are as expressed below:

$$
\begin{aligned}
& \Upsilon_{R 1}=\frac{E}{4 N_{0} N}\left\|\mathbf{X}_{R 1}^{\prime \prime}\right\|^{2}, \\
& \Upsilon_{R 2}=\frac{E}{4 N_{0} N}\left\|\mathbf{X}_{R 2}^{\prime \prime}\right\|^{2} .
\end{aligned}
$$

Case II is composed of two links which are a relay link ( $S$ $R 2-D)$ and an equivalent direct link $(R 1-D)$. The BER of the receiving signal at the destination terminal can be written as

$$
\begin{aligned}
P_{e}= & \left(1-Q\left(\sqrt{\Upsilon_{R 2}}\right)\right) Q\left(\sqrt{\Upsilon_{R 1}+\Upsilon_{R 2}}\right) \\
& +Q\left(\sqrt{\frac{\left(\Upsilon_{R 1}-\Upsilon_{R 2}\right)^{2}}{\Upsilon_{R 1}+\Upsilon_{R 2}}}\right) Q\left(\sqrt{\Upsilon_{R 2}}\right) .
\end{aligned}
$$

Using channel encoding including interleaving and the $(7,4)$ cyclic code, the $P_{e, \text { II }}$ can be derived as

$$
P_{e, \mathrm{II}}=\sum_{m=t+1}^{n} C_{n}^{m}\left(P_{e}\right)^{m}\left(1-P_{e}\right)^{n-m} .
$$

4.3. Case III. The occurrence of $R 3$ is like $R 2$, wherein the coming probability $P_{S 2}$ of event $S 2$ in the time interval $T$ of $50 \mathrm{~s}$ is

$$
P_{S 2}=1-\left.\exp \left(-\frac{\mathrm{t}}{\lambda}\right)\right|_{T=50, \lambda=180}=0.2425 .
$$

The communication model in Figure 4 can be considered to be composed of two basic models. One is marked with red lines, while the other is marked with blue lines. The principle of the red model is the same as that of the communication model in Figure 2 of which the SNR $\Upsilon_{R 1, R 2}$ at the destination terminal is written as

$$
\Upsilon_{R 1, R 2}=\frac{E}{4 N_{0} N}\left\|\mathbf{X}_{R 1, R 2}^{\prime \prime}\right\|^{2} .
$$

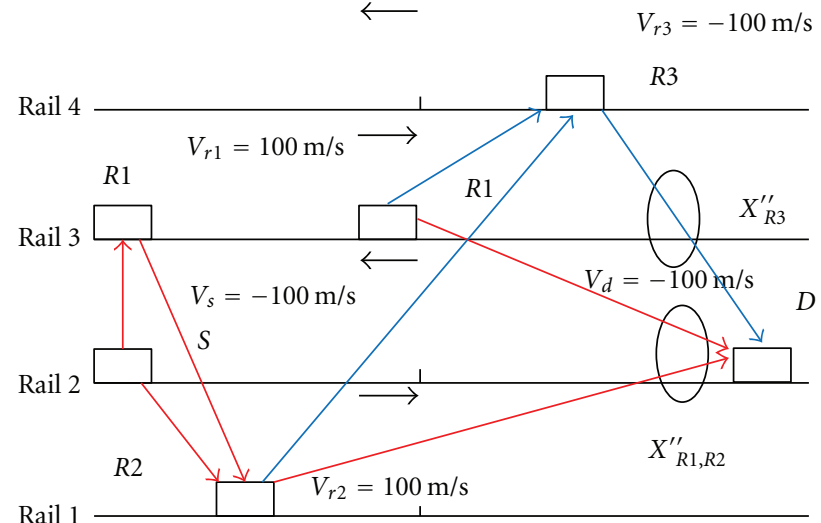

FIgUre 4: Communication model of Case III.

Then the red model can be considered equivalent to a direct link from the relay $R 1$ to the destination $D$. The $\mathbf{X}_{R 3}^{\prime \prime}$ is the estimation signals of the relay link $(R 1-R 3-D)$ at the destination, and the SNR $\Upsilon_{R 3}$ is

$$
\Upsilon_{R 3}=\frac{E}{4 N_{0} N}\left\|\mathbf{X}_{R 3}^{\prime \prime}\right\|^{2} .
$$

Combining the relay link with the direct link, the BER of the communication model in Figure 4 is

$$
\begin{gathered}
P_{e}=\left(1-Q\left(\sqrt{\Upsilon_{R 3}}\right)\right) Q\left(\sqrt{\Upsilon_{R 1, R 2}+\Upsilon_{R 3}}\right) \\
+Q\left(\sqrt{\left.\frac{\left(\Upsilon_{R 1, R 2}-\Upsilon_{R 3}\right)^{2}}{\Upsilon_{R 1, R 2}+\Upsilon_{R 3}}\right) Q\left(\sqrt{\Upsilon_{R 3}}\right),}\right. \\
P_{e, \text { III }}=\sum_{m=t+1}^{n} C_{n}^{m}\left(P_{e}\right)^{m}\left(1-P_{e}\right)^{n-m} .
\end{gathered}
$$

\section{Numerical Analysis}

5.1. Frequency Selection. The maximum propagation distance of the proposed train-to-train communication model is $6 \mathrm{Km}$. In order to avoid drastic signal attenuation of a long transmit distance, considering the Hata-Okumura suburban model [15], it is suitable to choose a frequency among the UHF band. The frequency determined at $300 \mathrm{MHz}$ and the change of the path loss of the signal with distance in a suburban area is presented in Figure 5. As shown in Figure 5, the path loss of the signal in a suburban area at the distance of $6 \mathrm{Km}$ is $-142.23 \mathrm{~dB}$.

5.2. Channel Model. The channel model used in this paper is the train-to-train suburban communication model in COST $207[16,17]$, which has six paths and whose maximum time delay is $5 \mu \mathrm{s}$. The speed of the high speed train is up to $100 \mathrm{~m} / \mathrm{s}$, so the Doppler frequency shift, in the range of $[-200,200] \mathrm{Hz}$, is added to each of six paths. The parameters of the suburban model in COST 207 are listed in Table 1.

5.3. Transmit Power. The minimum receiving level of the high-speed railway is $-92 \mathrm{dBm}$ [18], which is published 
TABLE 1: Parameters of the suburban model in COST 207.

\begin{tabular}{lcc}
\hline Number of path & Time delay $(\mu \mathrm{s})$ & Path power \\
\hline 1 & 0 & 1 \\
2 & 0.1 & 0.4 \\
3 & 0.2 & 0.16 \\
4 & 0.3 & 0.06 \\
5 & 0.4 & 0.03 \\
6 & 0.5 & 0.01 \\
\hline
\end{tabular}

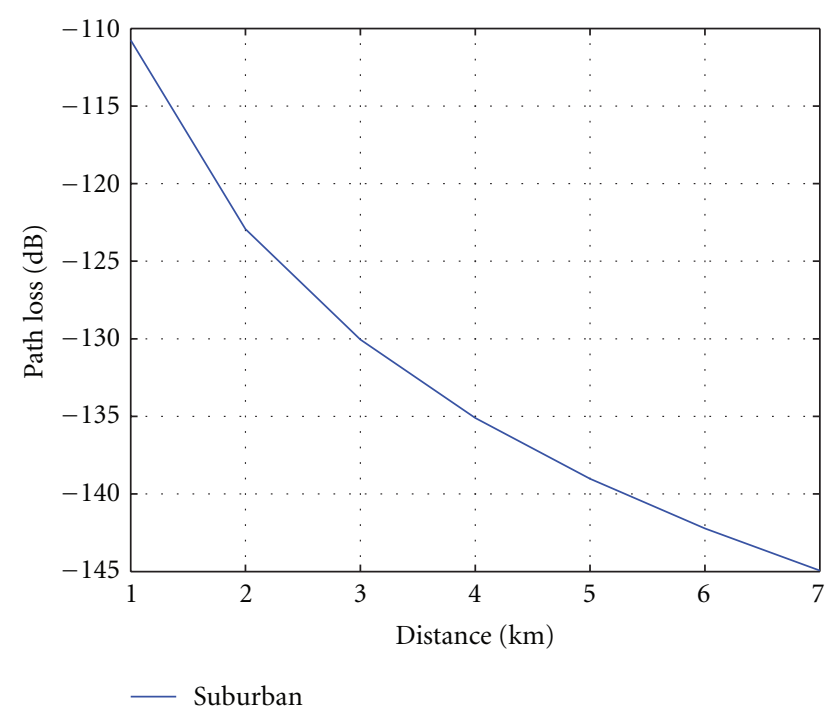

FIGURE 5: Change of the path loss of the signal with distance in suburban area.

by UIC. The required SNR for the best reception is $10 \mathrm{~dB}$ and the bandwidth of train-to-train communication is $1 \mathrm{MHz}$. For the longest communication distance, the path loss is $-142.23 \mathrm{~dB}$. Therefore the transmit power $P$ can be calculated by

$$
\begin{aligned}
P= & 10 \log _{10}(\mathrm{KTB})+\mathrm{SNR}-L_{\text {Path Loss }}+\sigma 2 \\
= & 10 \log _{10}\left(1.38 * 10^{-23} \frac{\mathrm{J}}{\mathrm{K}} * 290 \mathrm{~K} * 10^{6}\right) \\
& +10 \mathrm{~dB}+142.23 \mathrm{db}+3 \mathrm{~dB} \\
= & 11.25 \mathrm{dBW}(13.59 \mathrm{~W}) .
\end{aligned}
$$

As is shown in (23), the lowest transmit power of train-totrain communication is $13.59 \mathrm{~W}$.

5.4. Key Technique. The bit stream from the source is firstly mapped to the symbol with $\pi / 4$-QPSK and then the OFDM modulation is performed. For the OFDM, there are 1024 subcarriers with 512 mainly applied to transmit useful messages. The length of the cyclic prefix is 128 and the interval of the pilot is set to 5 . The bandwidth is $1 \mathrm{MHz}$.

\section{Simulation Result}

The train-to-train communication model performance is measured in terms of the BER by altering the SNR using the

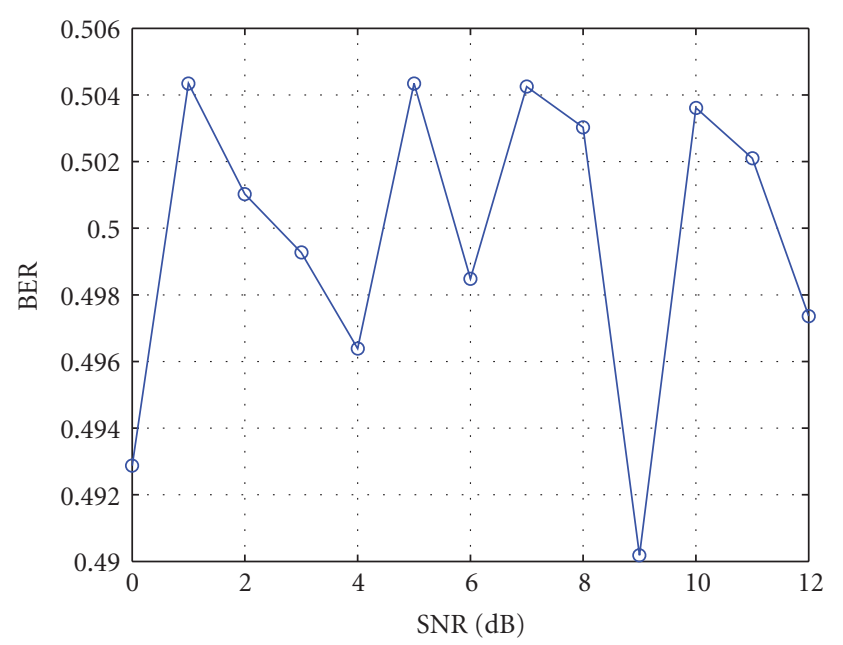

FIGURE 6: BER of two-train direct communication on the same track without multihop and cooperation.

Monte Carlo simulation. The results indicate that the trainto-train communication model works better with BER = $10^{-6}$ at $\mathrm{SNR}=10 \mathrm{~dB}$ which satisfies the requirements of the transmission of the train control and prewarning messages [19]. The simulation results of the proposed train-to-train communication model are listed below, and as a contrast, the BER of the two-train direct communication on the same track without multihop is also presented.

In Figure 6, two trains on the same track communicate with each other directly without any help from other trains. In this situation, we can see the BER fluctuates around 0.5, which does not ensure messages are transmitted correctly and may easily result in a severe railway traffic accident. This simulation result shows that it is necessary to introduce some trains as relays to help source trains propagate messages to destination trains.

Figure 7 presents the BER performance of train-to-train communication model under Case I, Case II, and Case III with SNR from $0 \mathrm{~dB}$ to $12 \mathrm{~dB}$. From Figure 7, Case II has better performance than Case I and Case III at a low SNR. When the SNR is above $4 \mathrm{~dB}$, the performance of Case II is comparable to that of Case III, but is much better than Case III until SNR $=8 \mathrm{~dB}$. It can be concluded that at a low SNR the possible relay $R 1$ and $R 2$ are able to ensure accurate transmission of the control and prewarning messages.

From the derivation above, the BERs $P_{e, \mathrm{II}}, P_{e, \mathrm{I}}$, and $P_{e, \mathrm{III}}$ of Case I, Case II, and Case III are written as

$$
\begin{aligned}
P_{e, \mathrm{I}}=\sum_{m=t+1}^{n} C_{n}^{m}\left(Q\left(\sqrt{2 \Upsilon_{R 1}}\right)+Q\left(\sqrt{2 \Upsilon_{D}}\right)\right. \\
\left.-2 Q\left(\sqrt{2 \Upsilon_{R 1}}\right) Q\left(\sqrt{2 \Upsilon_{D}}\right)\right)^{m} \\
\times\left(1-Q\left(\sqrt{2 \Upsilon_{R 1}}\right)+Q\left(\sqrt{2 \Upsilon_{D}}\right)\right. \\
\left.-2 Q\left(\sqrt{2 \Upsilon_{R 1}}\right) Q\left(\sqrt{2 \Upsilon_{D}}\right)\right)^{n-m}
\end{aligned}
$$




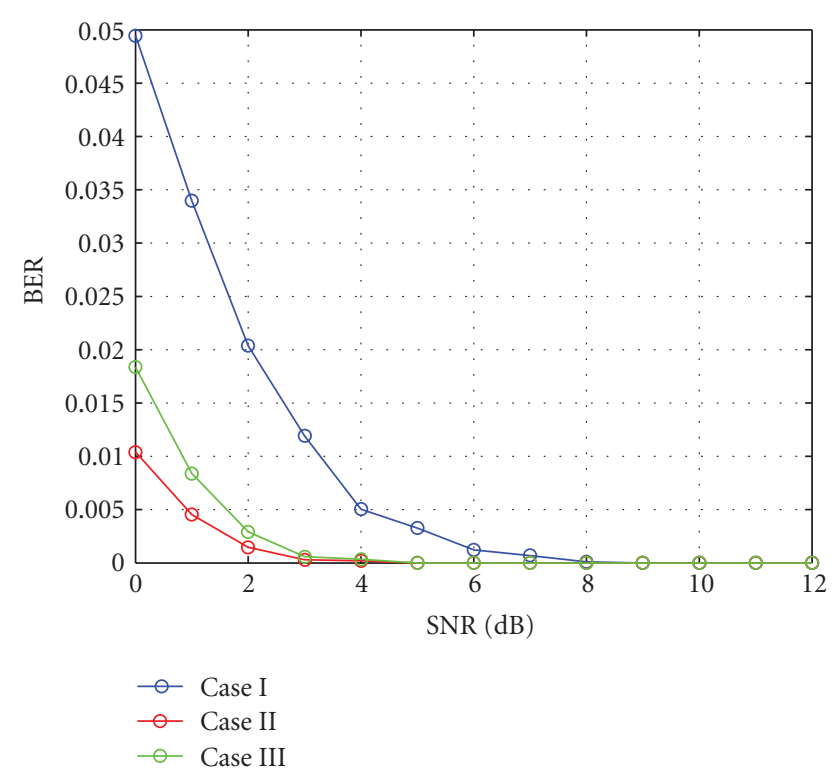

FIgure 7: BER of Case I, Case II, and Case III of train-to-train communication model.

$$
\begin{aligned}
& P_{e, \mathrm{II}}=\sum_{m=t+1}^{n} C_{n}^{m}\left(\left(1-Q\left(\sqrt{\Upsilon_{R 2}}\right)\right) Q\left(\sqrt{\Upsilon_{R 1}+\Upsilon_{R 2}}\right)\right. \\
& \left.+Q\left(\sqrt{\frac{\left(\Upsilon_{R 1}-\Upsilon_{R 2}\right)^{2}}{\Upsilon_{R 1}+\Upsilon_{R 2}}}\right) Q\left(\sqrt{\Upsilon_{R 2}}\right)\right)^{m} \\
& \times\left(1-\left(1-Q\left(\sqrt{\Upsilon_{R 2}}\right)\right) Q\left(\sqrt{\Upsilon_{R 1}+\Upsilon_{R 2}}\right)\right. \\
& \left.+\mathrm{Q}\left(\sqrt{\frac{\left(\Upsilon_{R 1}-\Upsilon_{R 2}\right)^{2}}{\Upsilon_{R 1}+\Upsilon_{R 2}}}\right) \mathrm{Q}\left(\sqrt{\Upsilon_{R 2}}\right)\right)^{n-m}, \\
& P_{e, \text { III }}=\sum_{m=t+1}^{n} C_{n}^{m}\left(\left(1-Q\left(\sqrt{\Upsilon_{R 3}}\right)\right) Q\left(\sqrt{\Upsilon_{R 1, R 2}+\Upsilon_{R 3}}\right)\right. \\
& \left.+Q\left(\sqrt{\frac{\left(\Upsilon_{R 1, R 2}-\Upsilon_{R 3}\right)^{2}}{\Upsilon_{R 1, R 2}+\Upsilon_{R 3}}}\right) Q\left(\sqrt{\Upsilon_{R 3}}\right)\right)^{m} \\
& \times\left(1-\left(1-Q\left(\sqrt{\Upsilon_{R 3}}\right)\right) Q\left(\sqrt{\Upsilon_{R 1, R 2}+\Upsilon_{R 3}}\right)\right. \\
& \left.+Q\left(\sqrt{\frac{\left(\Upsilon_{R 1, R 2}-\Upsilon_{R 3}\right)^{2}}{\Upsilon_{R 1, R 2}+\Upsilon_{R 3}}}\right) Q\left(\sqrt{\Upsilon_{R 3}}\right)\right)^{n-m} .
\end{aligned}
$$

Supposing SNR $=10 \mathrm{~dB}$, the theoretical value of the BERs under Case I, Case II, and Case III all approach zero, which corresponds to the simulation result in Figure 6.

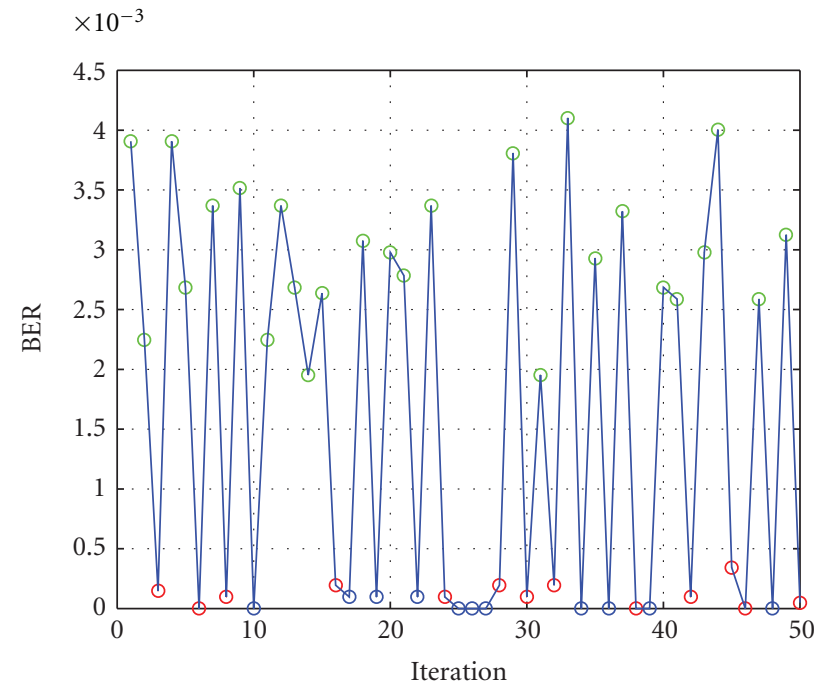

FIGURE 8: BER of train-to-train communication model SNR $=5 \mathrm{~dB}$ based on the Poisson process.

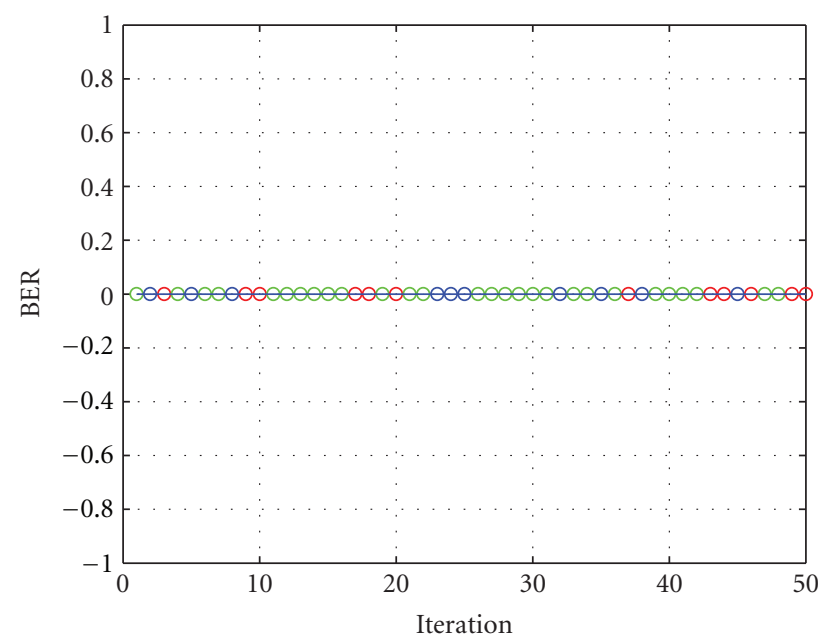

FIGURE 9: BER of train-to-train communication model at at SNR = $10 \mathrm{~dB}$ based on the Poisson process.

Figures 8 and 9 describe the BER performance of the train-to-train communication model based on the Poisson process when SNR $=5 \mathrm{~dB}$ and $10 \mathrm{~dB}$. Using the Monte Carlo method, the simulation is repeated 50 times. At each time, the topology of possibly-occurring relays is different. In these figures, the blue dots represent Case I, while the red and green dots represent Case II and Case III, respectively. At SNR $=0 \mathrm{~dB}$, the BERs of Case I, Case II, and Case III are all maintained at about $10^{-2}$. At SNR $=5 \mathrm{~dB}$, the BER of Case I declines to $10^{-3}$, and particularly the BERs of Case II and Case III reach $10^{-4}$. When SNR $=10 \mathrm{~dB}$, the BER is basically maintained at 0 in 50 Monte Carlo simulations. It is proved that at SNR $=10 \mathrm{~dB}$ the reliability of the train-totrain communication model is sufficient to satisfy the needs of safety of the high-speed railway.

According to the analysis above, the train-to-train communication has some advantages over the GSM-R and RCAS 
TABLE 2: Comparison of technique index of the GSM-R, RCAS, and train-to-train in physical layer.

\begin{tabular}{lccc}
\hline Technique index & GSM-R & RCAS & Train-to-Train \\
\hline $\begin{array}{l}\text { Bandwidth } \\
(\mathrm{MHz} / \mathrm{W})\end{array}$ & $10^{-2}$ & 10 & 0.067 \\
$\begin{array}{l}\text { Minimum receiving } \\
\text { SNR } \\
(\mathrm{dB} / \mathrm{MHz} / \mathrm{W})\end{array}$ & 3.32 & 2.5 & 0.66 \\
$\begin{array}{l}\text { Average BER } \\
(/ \mathrm{MHz} / \mathrm{W})\end{array}$ & $2.5 * 10^{-6}$ & $10^{-4}$ & $7.4 * 10^{-8}$ \\
$\begin{array}{l}\text { Transmit power } \\
\text { (W/MHz) }\end{array}$ & 40 & 0.1 & 13.59 \\
$\begin{array}{l}\text { Coverage area } \\
\text { (Km) }\end{array}$ & 3 & 5 & 6 \\
$\begin{array}{l}\text { Minimum delay } \\
(\mathrm{s})\end{array}$ & 1 & 1 & 10 \\
$\begin{array}{l}\text { Frequency } \\
(\mathrm{MHz})\end{array}$ & 900 & 460 & 300 \\
\hline
\end{tabular}

in the physical layer, such as minimum receiving SNR, average BER, and coverage area, which are listed in Table 2 $[4-7,18]$.

Admittedly, the minimum time delay of the train-totrain communication model is longer than that of the GSM$\mathrm{R}$. The minimum time delay of train-to-train communication model is $10 \mathrm{~s}$. However, as a subsidiary role, the duty of the train-to-train communication model is to transmit the prewarning messages accurately when the existing train control system is malfunctioning. Once the front train is stopping on the rail track because of an accident, after $10 \mathrm{~s}$ ' message transmit delay, the remaining distance $S$ between neighboring trains on the same rail track can be calculated as

$$
S=10 \mathrm{Km}-\left(360 \mathrm{Km} / \mathrm{h} * \frac{10 \mathrm{~s}}{3600}\right)=9 \mathrm{Km} .
$$

The braking distance of a high-speed train is about $5 \mathrm{~km}$ at the speed of $300 \mathrm{~km} / \mathrm{h}$ [20]. As seen from (25), the remaining $9 \mathrm{~km}$ is sufficient for the drivers to decide how to deal with the emergency. That is, the time delay of $10 \mathrm{~s}$ absolutely does not affect the following processes and reaction to the prewarning messages of the following trains on the same track.

\section{Conclusion}

This paper proposes a novel multihop train-to-train communication model using $300 \mathrm{MHz}$ based on the Poisson process in the scenario of a high-speed railway, introducing OFDM and MIMO. The BER of the train-to-train model is decreased to $10^{-6}$ when SNR is $10 \mathrm{~dB}$, and the minimum receiving level of this model is $-84 \mathrm{dBm}$ corresponding to the standards established by UIC in a high-speed railway scenario. In contrast to the GSM-R and RCAS, the trainto-train communication model has advantages in minimum receiving SNR, average BER, and coverage area in the physical layer, which ensures accurate transmission of the control and prewarning messages.

\section{Acknowledgments}

This research was supported by the Program for New Century Excellent Talents in University under Grant no. NCET-09-0206, Fundamental Research Funds for the Central Universities under Grant no. 2010JBZ008 and no. 2011YJS010, Key Project of State Key Lab under Grant no. RCS2011ZZ008, National Natural Science Foundation of China under Grant no. 60830001, Beijing Natural Science Foundation under Grant no. 4112048, and Program for Changjiang Scholars and Innovative Research Team in University under Grant no. IRT0949.

\section{References}

[1] P. Y. Liu, B. Ai, and Z. D. Zhong, "A message broadcast model for train-to-train communication network," Information-An International Interdisciplinary Journal. In press.

[2] "Accidents in Deciding Frequency by Cause," http://safetydata .fra.dot.gov/officeofsafety/publicsite/Query/inctallyl.aspx.

[3] C. R. Garcia, A. Lehner, and T. Strang, "A broadcast vehicleto-vehicle communication system in railway environments," in Proceedings of the 1st Annual International Symposium on Vehicular Computing Systems, 2008.

[4] T. Strang, M. M. zu Hörste, and X. G. Gu, "A railway collision avoidance system exploiting ad-hoc inter-vehicle communications and GALILEO," in Proceedings of the 13th World Congress and Exhibition on Intelligent Transportation Systems and Services (ITS '06), London, UK, 2006, http://citeseerx.ist.psu.edu/ viewdoc/summary?doi=10.1.1.140.4267.

[5] A. Lehner, C. Rico-García, E. Wige, and T. Strang, "A multibroadcast communication system for high dynamic vehicular ad-hoc networks," in Proceedings of the International Conference on Ultra Modern Telecommunications and Workshops (ICUMT '09), pp. 1-6, October 2009.

[6] C. R. García, A. Lehner, T. Strang, and M. Röckl, "Comparison of collision avoidance systems and applicability to rail transport," in Proceedings of the 7th International Conference on Intelligent Transport Systems Telecommunications (ITST '07), pp. 1-6, June 2007.

[7] C. R. García, A. Lehner, T. Strang, and K. Frank, "Channel model for train to train communication using the $400 \mathrm{MHz}$ band," in Proceedings of the IEEE 67th Vehicular Technology Conference-Spring (VTC '08), pp. 3082-3086, May 2008.

[8] A. Lehner, C. Rico García, T. Strang, and O. Heirich, "Measurement and analysis of the direct train to train propagation channel in the $70 \mathrm{~cm}$ UHF-band," in Proceedings of the $3 \mathrm{rd}$ International Workshop on Communication Technologies for Vehicles, vol. 6569, pp. 45-57, 2011.

[9] P. Zhao, The Operation and Reorganization of the High Speed Train, China Railway Publishing House, 2009.

[10] J. M. Zhang and B. M. Han, "Analysis and calculation of the scheduled waiting time for the train route," in Proceedings of the 4th International Conference on Intelligent Computation Technology and Automation (ICICTA '11), vol. 1, pp. 54-58, March 2011.

[11] S. M. Ross, Stochastic Process, John Wiley \& Sons, New York, NY, USA, 1996.

[12] http://news.xinhuanet.com/fortune/2011-07/01/c_121609685 .htm. 
[13] K. J. Ray Liu, A. K. Sadek, W. F. Su, and A. Kwasinski, Cooperative Communications and Networking, Publishing House of Electronics Industry, 2010.

[14] J. G. Proakis, Digital Communication, McGraw-Hill, London, UK.

[15] H. B. Cheng and Y. D. Yao, "Power adaptation for multihop networks with end-to-end BER requirements," IEEE Transactions on Vehicular Technology, vol. 59, no. 7, pp. 35453554, 2010.

[16] M. Patzold, Mobile Fading Channels, John Wiley \& Sons, New York, NY, USA, 2002.

[17] L. Xiong, X. Lu, Z. Zhong, B. Ai, and M. Zhu, "Simulation on the characteristics of the wireless channel for high-speed railway GSM-R system," China Railway Science, vol. 31, no. 5, pp. 84-89, 2010.

[18] GSM-R QoS Working Group, O-2475 ERTMS/GSM-R Quality of Service Test Specification, UIC, Paris, Farnce, 2006.

[19] Z. D. Zhong, B. Ai, and Q. Y. Liu, Applications of Fundamental Theory of Railway Digital Mobile Communication System (GSM), TsingHua University Press, 2009.

[20] Z. M. Wu, J. Y. ZUO, and C. Tian, "Discussion on the design parameter of emergency brake distance of high-speed train," Electric Locomotives \& Mass Transit Vehicles, vol. 32, no. 4, pp. $1-4,2009$. 

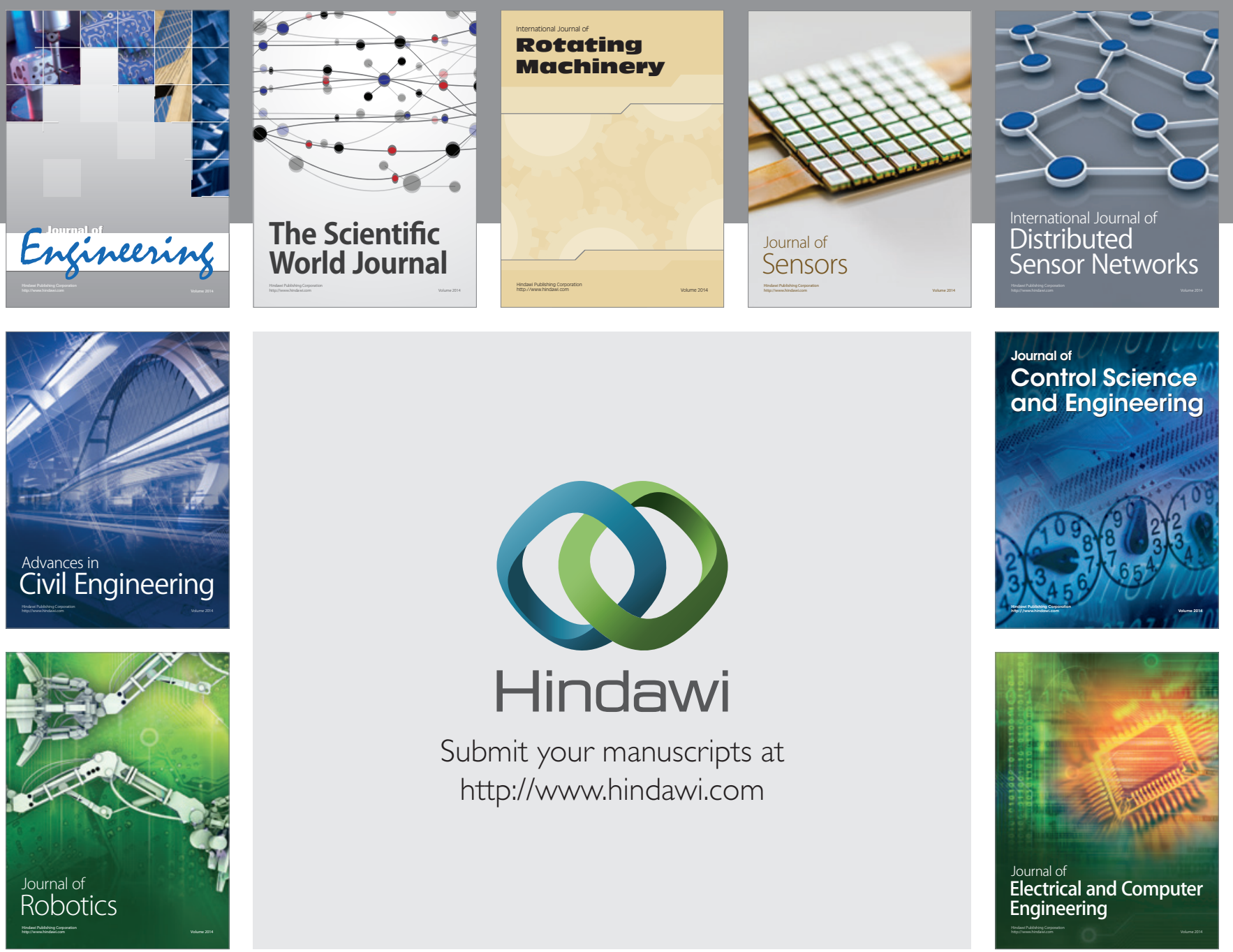

Submit your manuscripts at

http://www.hindawi.com
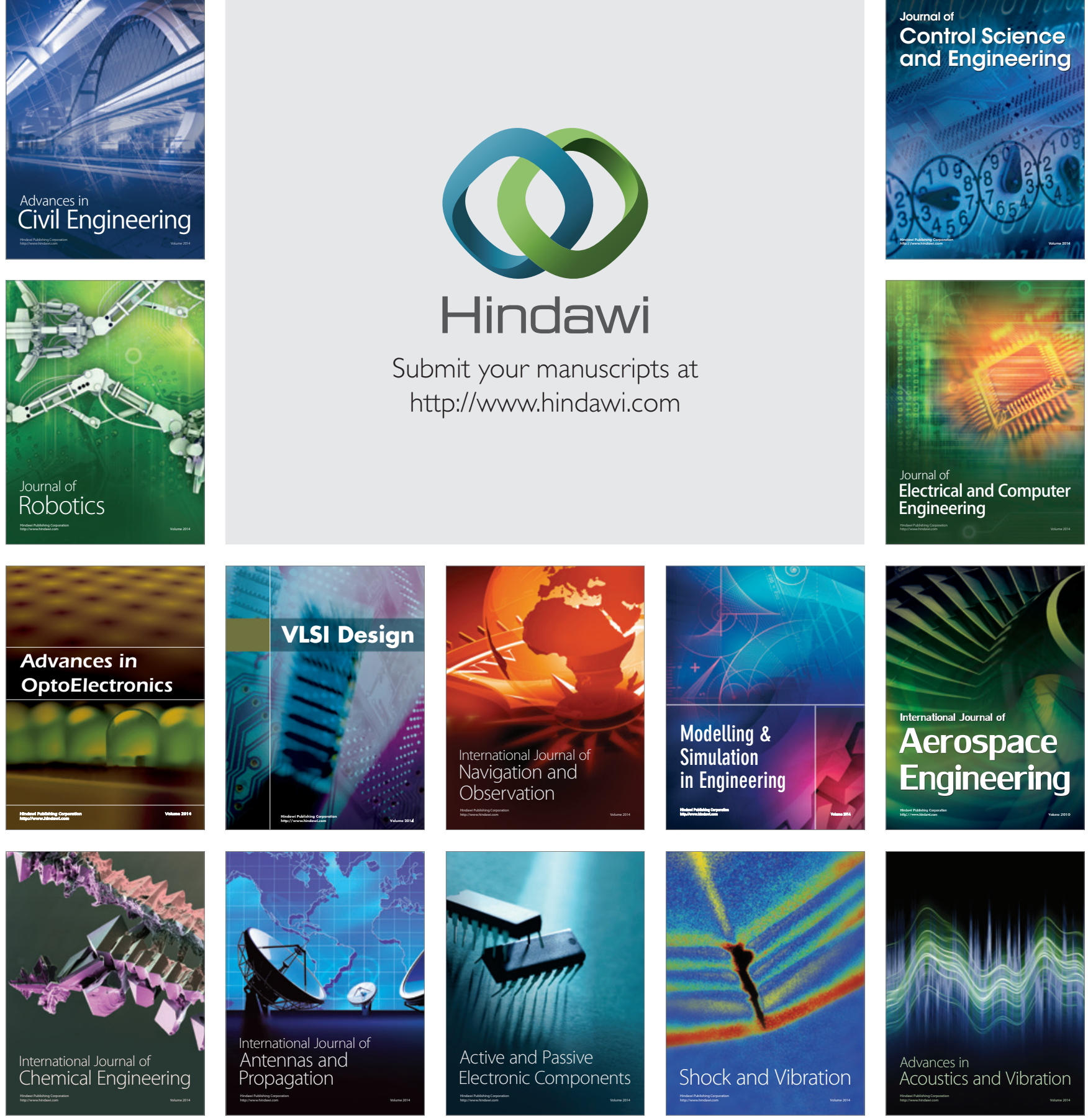